Article

\title{
Some Types of Subsemigroups Characterized in Terms of Inequalities of Generalized Bipolar Fuzzy Subsemigroups
}

\author{
Pannawit Khamrot ${ }^{1}$ (D) and Manoj Siripitukdet ${ }^{1,2, *}$ \\ 1 Departament of Mathematics, Faculty of Science, Naresuan University, Phitsanulok 65000, Thailand; \\ pannawit.k@gmail.com \\ 2 Research Center for Academic Excellence in Mathematics, Faculty of Science, Naresuan University, \\ Phitsanulok 65000, Thailand \\ * Correspondence: manojs@nu.ac.th
}

Received: 14 November2017; Accepted: 23 November 2017; Published: 27 November 2017

\begin{abstract}
In this paper, we introduce a generalization of a bipolar fuzzy (BF) subsemigroup, namely, a $\left(\alpha_{1}, \alpha_{2} ; \beta_{1}, \beta_{2}\right)$-BF subsemigroup. The notions of $\left(\alpha_{1}, \alpha_{2} ; \beta_{1}, \beta_{2}\right)$-BF quasi(generalized bi-, bi-) ideals are discussed. Some inequalities of $\left(\alpha_{1}, \alpha_{2} ; \beta_{1}, \beta_{2}\right)$-BF quasi(generalized bi-, bi-) ideals are obtained. Furthermore, any regular semigroup is characterized in terms of generalized BF semigroups.
\end{abstract}

Keywords: generalized bipolar fuzzy (BF) semigroup; $\left(\alpha_{1}, \alpha_{2} ; \beta_{1}, \beta_{2}\right)$-bipolar fuzzy subsemigroup; fuzzy quasi(generalized bi-, bi-) ideal; regular semigroup

\section{Introduction}

Most of the bipolarities separate positive and negative information response; positive information representations are compiled to be possible, while negative information representations are impossible [1]. The bipolar information of evaluation can help to evaluate decisions. Sometimes, decisions are not only influenced by the positive decision criterion, but also with the negative decision criterion, for example, environmental and social impact assessment. Evaluated alternative consideration should weigh the negative effects to select the optimal choice. Therefore bipolar information affects the effectiveness and efficiency of decision making. It is used in decision-making problems, organization problems, economic problems, evaluation, risk management, environmental and social impact assessment, and so forth. Thus, the concept of bipolar fuzzy (BF) sets are more relevant in mathematics.

In 1965, Zadeh [2] introduced the fuzzy set theory, which can be applied to many areas, such as mathematics, statistics, computers, electrical instruments, the industrial industry, business, engineering, social applications, and so forth. In 2003, Bucolo et al. [3] proposed small-world networks of fuzzy chaotic oscillators. The fuzzy set was used to establish the mathematical method for dealing with imprecise and uncertain environments. In 1971, Rosenfeld [4] applied fuzzy sets to group structures. Then, the fuzzy set was used in the theory of semigroups in 1979. Kuroki [5] initiated fuzzy semigroups based on the notion of fuzzy ideals in semigroups and introduced some properties of fuzzy ideals and fuzzy bi-ideals of semigroups. The fundamental concepts of BF sets were initiated by Zhang [6] in 1994. He innovated the BF set as BF logic, which has been widely applied to solve many real-world problems. In 2000, Lee [7] studied the notion of bipolar-valued fuzzy sets. Kim et al. [8] studied the notions of BF subsemigroups, BF left (right, bi-) ideals. He provided some necessary and sufficient conditions for a BF subsemigroup and BF left (right, bi-) ideals of semigroups.

In this paper, generalizations of BF semigroups are introduced. Definitions and properties of $\left(\alpha_{1}, \alpha_{2} ; \beta_{1}, \beta_{2}\right)$-BF quasi (generalized bi-, bi-) ideals are obtained. Some inequalities of $\left(\alpha_{1}, \alpha_{2} ; \beta_{1}, \beta_{2}\right)$-BF 
quasi (generalized bi-, bi-) ideals are obtained. Finally, we characterize a regular semigroup in terms of generalized BF semigroups.

\section{Preliminaries}

In this section, we give definitions and examples that are used in this paper. By a subsemigroup of a semigroup $S$ we mean a non-empty subset $A$ of $S$ such that $A^{2} \subseteq A$, and by a left (right) ideal of $S$ we mean a non-empty subset $A$ of $S$ such that $S A \subseteq A(A S \subseteq A)$. By a two-sided ideal or simply an ideal, we mean a non-empty subset of a semigroup $S$ that is both a left and a right ideal of $S$. A non-empty subset $A$ of $S$ is called an interior ideal of $S$ if $S A S \subseteq A$, and a quasi-ideal of $S$ if $A S \cap S A \subseteq A$. A subsemigroup $A$ of $S$ is called a bi-ideal of $S$ if $A S A \subseteq A$. A non-empty subset $A$ is called a generalized bi-ideal of $S$ if $A S A \subseteq A$ [9].

By the definition of a left (right) ideal of a semigroup $S$, it is easy to see that every left (right) ideal of $S$ is a quasi-ideal of $S$.

Definition 1. A semigroup $S$ is called regular if for all $a \in S$ there exists $x \in S$ such that $a=$ axa.

Theorem 1. For a semigroup $S$, the following conditions are equivalent.

(1) $S$ is regular.

(2) $R \cap L=R L$ for every right ideal $R$ and every left ideal $L$ of $S$.

(3) $A S A=A$ for every quasi-ideal $A$ of $S$.

Definition 2. Let $X$ be a set; a fuzzy set (or fuzzy subset) $f$ on $X$ is a mapping $f: X \rightarrow[0,1]$, where $[0,1]$ is the usual interval of real numbers.

The symbols $f \wedge g$ and $f \vee g$ will denote the following fuzzy sets on $S$ :

$$
\begin{aligned}
& (f \wedge g)(x)=f(x) \wedge g(x) \\
& (f \vee g)(x)=f(x) \vee g(x)
\end{aligned}
$$

for all $x \in S$.

A product of two fuzzy sets $f$ and $g$ is denoted by $f \circ g$ and is defined as

$$
(f \circ g)(x)= \begin{cases}\bigvee_{x=y z}\{f(y) \wedge g(z)\}, & \text { if } x=y z \text { for some } y, z \in S \\ 0, & \text { otherwise }\end{cases}
$$

Definition 3. Let $S$ be a non-empty set. A BF set $f$ on $S$ is an object having the following form:

$$
f:=\left\{\left(x, f_{p}(x), f_{n}(x)\right): x \in S\right\}
$$

where $f_{p}: S \rightarrow[0,1]$ and $f_{n}: S \rightarrow[-1,0]$.

Remark 1. For the sake of simplicity we use the symbol $f=\left(S ; f_{p}, f_{n}\right)$ for the BF set $f=\left\{\left(x, f_{p}(x), f_{n}(x)\right): x \in S\right\}$.

Definition 4. Given a BF set $f=\left(S ; f_{p}, f_{n}\right), \alpha \in[0,1]$ and $\beta \in[-1,0]$, the sets

$$
P(f ; \alpha):=\left\{x \in S \mid f_{p}(x) \geq \alpha\right\}
$$

and

$$
N(f ; \beta):=\left\{x \in S \mid f_{n}(x) \leq \beta\right\}
$$


are called the positive $\alpha$-cut and negative $\beta$-cut of $f$, respectively. The set $C(f ;(\alpha, \beta)):=P(f ; \alpha) \cap N(f ; \beta)$ is called the bipolar $(\alpha, \beta)$-cut of $f$.

We give the generalization of a BF subsemigroup, which is defined by Kim et al. (2011).

Definition 5. $A$ BF set $f=\left(S ; f_{p}, f_{n}\right)$ on $S$ is called a $\left(\alpha_{1}, \alpha_{2} ; \beta_{1}, \beta_{2}\right)$-BF subsemigroup on $S$, where $\alpha_{1}, \alpha_{2} \in[0,1], \beta_{1}, \beta_{2} \in[-1,0]$ if it satisfies the following conditions:

(1) $f_{p}(x y) \vee \alpha_{1} \geq f_{p}(x) \wedge f_{p}(y) \wedge \alpha_{2}$

(2) $f_{n}(x y) \wedge \beta_{2} \leq f_{n}(x) \vee f_{n}(y) \vee \beta_{1}$

for all $x, y \in S$.

We note that every BF subsemigroup is a $(0,1,-1,0)$-BF subsemigroup.

The following examples show that $f=\left(S ; f_{p}, f_{n}\right)$ is a $\left(\alpha_{1}, \alpha_{2} ; \beta_{1}, \beta_{2}\right)$-BF subsemigroup on $S$ but $f=\left(S ; f_{p}, f_{n}\right)$ is not a BF subsemigroup on $S$.

Example 1. The set $S=\{2,3,4, \ldots\}$ is a semigroup under the usual multiplication. Let $f=\left(S ; f_{p}, f_{n}\right)$ be a BF set on $S$ defined as follows:

$$
f_{p}(x):=\frac{1}{x+2} \text { and } f_{n}(x):=-\frac{1}{x+2}
$$

for all $x \in S$.

Let $x, y \in S$. Then

$$
f_{p}(x y)=\frac{1}{x y+2}<\frac{1}{x+2}=f_{p}(x)
$$

and

$$
f_{p}(x y)=\frac{1}{x y+2}<\frac{1}{y+2}=f_{p}(y)
$$

Thus, $f_{p}(x y)<f_{p}(x) \wedge f_{p}(y)$. Therefore $f=\left(S ; f_{p}, f_{n}\right)$ is not a BF subsemigroup on $S$.

Let $\alpha_{2} \in[0,1], \beta_{1} \in[-1,0], \alpha_{1}=\frac{1}{4}$ and $\beta_{2}=-\frac{1}{4}$. Thus for all $x, y \in S$,

$$
f_{p}(x y) \vee \frac{1}{4} \geq \frac{1}{x+2} \wedge \frac{1}{y+2} \geq f_{p}(x) \wedge f_{p}(y) \wedge \alpha_{2}
$$

and

$$
f_{n}(x y) \wedge-\frac{1}{4} \leq-\frac{1}{x+2} \vee-\frac{1}{y+2} \leq f_{n}(x) \vee f_{n}(y) \vee \beta_{1}
$$

Hence $f=\left(S ; f_{p}, f_{n}\right)$ is a $\left(\frac{1}{4}, \alpha_{2} ; \beta_{1},-\frac{1}{4}\right)-B F$ subsemigroup on $S$.

We note that $f=\left(S ; f_{p}, f_{n}\right)$ is a $\left(\alpha_{1}, \alpha_{2} ; \beta_{1}, \beta_{2}\right)$-BF subsemigroup on $S$ for all $\alpha_{1} \geq \frac{1}{4}$ and $\beta_{2} \leq-\frac{1}{4}$.

Definition 6. $A$ BF set $f=\left(S ; f_{p}, f_{n}\right)$ on $S$ is called $a\left(\alpha_{1}, \alpha_{2} ; \beta_{1}, \beta_{2}\right)$-BF left (right) ideal on $S$, where $\alpha_{1}, \alpha_{2} \in[0,1]$, and $\beta_{1}, \beta_{2} \in[-1,0]$ if it satisfies the following conditions:

(1) $f_{p}(x y) \vee \alpha_{1} \geq f_{p}(y) \wedge \alpha_{2}\left(f_{p}(x y) \vee \alpha_{1} \geq f_{p}(x) \wedge \alpha_{2}\right)$

(2) $f_{n}(x y) \wedge \beta_{2} \leq f_{n}(y) \vee \beta_{1}\left(f_{n}(x y) \wedge \beta_{2} \leq f_{n}(x) \vee \beta_{1}\right)$

for all $x, y \in S$.

A BF set $f=\left(S ; f_{p}, f_{n}\right)$ on $S$ is called a $\left(\alpha_{1}, \alpha_{2} ; \beta_{1}, \beta_{2}\right)$-BF ideal on $S\left(\alpha_{1}, \alpha_{2} \in[0,1], \beta_{1}, \beta_{2} \in[-1,0]\right)$ if it is both a $\left(\alpha_{1}, \alpha_{2} ; \beta_{1}, \beta_{2}\right)$-BF left ideal and a $\left(\alpha_{1}, \alpha_{2} ; \beta_{1}, \beta_{2}\right)$-BF right ideal on $S$.

By Definition 6, every $\left(\alpha_{1}, \alpha_{2} ; \beta_{1}, \beta_{2}\right)$-BF ideal on a semigroup $S$ is a $\left(\alpha_{1}, \alpha_{2} ; \beta_{1}, \beta_{2}\right)$-BF subsemigroup on $S$.

We note that a $(0,1,-1,0)$-BF left (right) ideal is a BF left (right) ideal. 
Definition 7. $A\left(\alpha_{1}, \alpha_{2} ; \beta_{1}, \beta_{2}\right)$-BF subsemigroup $f=\left(S ; f_{p}, f_{n}\right)$ on a subsemigroup $S$ is called $a\left(\alpha_{1}, \alpha_{2} ; \beta_{1}, \beta_{2}\right)$-BF bi-ideal on $S$, where $\alpha_{1}, \alpha_{2} \in[0,1]$, and $\beta_{1}, \beta_{2} \in[-1,0]$ if it satisfies the following conditions:

(1) $f_{p}($ xay $) \vee \alpha_{1} \geq f_{p}(x) \wedge f_{p}(y) \wedge \alpha_{2}$

(2) $f_{n}($ xay $) \wedge \beta_{2} \leq f_{n}(x) \vee f_{n}(y) \vee \beta_{1}$

for all $x, y \in S$.

We note that every $\left(\alpha_{1}, \alpha_{2} ; \beta_{1}, \beta_{2}\right)$-BF bi-ideal on a semigroup is a $\left(\alpha_{1}, \alpha_{2} ; \beta_{1}, \beta_{2}\right)$-BF subsemigroup on the semigroup.

\section{Generalized Bi-Ideal and Quasi-Ideal}

In this section, we introduce a product of $\mathrm{BF}$ sets and characterize a regular semigroup by generalized BF subsemigroups.

We let $f=\left(S: f_{p}, f_{n}\right)$ and $g=\left(S: g_{p}, g_{n}\right)$ be two BF sets on a semigroup $S$ and let $\alpha_{1}, \alpha_{2} \in[0,1]$, and $\beta_{1}, \beta_{2} \in[-1,0]$. We define two fuzzy sets $f_{p}^{\left(\alpha_{1}, \alpha_{2}\right)}$ and $f_{n}^{\left(\beta_{1}, \beta_{2}\right)}$ on $S$ as follows:

$$
\begin{aligned}
& f_{p}^{\left(\alpha_{1}, \alpha_{2}\right)}(x)=\left(f_{p}(x) \wedge \alpha_{1}\right) \vee \alpha_{2} \\
& f_{n}^{\left(\beta_{1}, \beta_{2}\right)}(x)=\left(f_{n}(x) \vee \beta_{2}\right) \wedge \beta_{1}
\end{aligned}
$$

for all $x \in S$.

We define two operations $\stackrel{\left(\alpha_{1}, \alpha_{2}\right)}{\wedge}$ and $\underset{\left(\beta_{1}, \beta_{2}\right)}{\vee}$ on $S$ as follows:

$$
\begin{aligned}
& \left(f_{p} \stackrel{\left(\alpha_{1}, \alpha_{2}\right)}{\wedge} g_{p}\right)(x)=\left(\left(f_{p} \wedge g_{p}\right)(x) \wedge \alpha_{1}\right) \vee \alpha_{2} \\
& \left(f_{n} \underset{\left(\beta_{1}, \beta_{2}\right)}{\wedge} g_{n}\right)(x)=\left(\left(f_{n} \vee g_{n}\right)(x) \vee \alpha_{2}\right) \wedge \alpha_{1}
\end{aligned}
$$

for all $x \in S$, and we define products $f_{p} \stackrel{\left(\alpha_{1}, \alpha_{2}\right)}{\circ} g_{p}$ and $f_{n} \underset{\left(\beta_{1}, \beta_{2}\right)}{\circ} g_{n}$ as follows:

For all $x \in S$,

$$
\begin{aligned}
& \left(f_{p} \stackrel{\left(\alpha_{1}, \alpha_{2}\right)}{\circ} g_{p}\right)(x)=\left(\left(f_{p} \bar{\circ} g_{p}\right)(x) \wedge \alpha_{1}\right) \vee \alpha_{2} \\
& \left(f_{n} \underset{\left(\beta_{1}, \beta_{2}\right)}{\circ} g_{n}\right)(x)=\left(\left(f_{n} \circ g_{n}\right)(x) \vee \alpha_{2}\right) \wedge \alpha_{1}
\end{aligned}
$$

where

$$
\begin{aligned}
& \left(f_{p} \bar{\circ} g_{p}\right)(x)= \begin{cases}\bigvee_{x=y z}\left\{f_{p}(y) \wedge g_{p}(z)\right\} & \text { if } x=y z \text { for some } y, z \in S \\
0 & \text { otherwise }\end{cases} \\
& \left(f_{n} \circ g_{n}\right)(x)= \begin{cases}\bigwedge_{y=y z}\left\{f_{n}(y) \vee g_{n}(z)\right\} & \text { if } x=y z \text { for some } y, z \in S \\
0 & \text { otherwise }\end{cases}
\end{aligned}
$$

We set

$$
f \underset{\left(\beta_{1}, \beta_{2}\right)}{\left(\alpha_{1}, \alpha_{2}\right)} g:=\left(S ; f_{p} \stackrel{\left(\alpha_{1}, \alpha_{2}\right)}{\circ} g_{p}, f_{n} \underset{\left(\beta_{1}, \beta_{2}\right)}{\circ} g_{n}\right)
$$

Then it is a BF set.

We note that

(1) $f_{p}^{(1,0)}(x)=f_{p}(x)$,

(2) $f_{n}^{(0,-1)}(x)=f_{n}(x)$, 
(3) $\quad f=\left(S ; f_{p}, f_{n}\right)=\left(S ; f_{p}^{(1,0)}, f_{n}^{(0,-1)}\right)$,

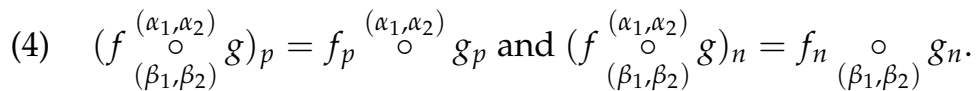

Definition 8. A BF set $f=\left(S ; f_{p}, f_{n}\right)$ on $S$ is called a $\left(\alpha_{1}, \alpha_{2} ; \beta_{1}, \beta_{2}\right)$-BF generalized bi-ideal on $S$, where $\alpha_{1}, \alpha_{2} \in[0,1]$, and $\beta_{1}, \beta_{2} \in[-1,0]$ if it satisfies the following conditions:

(1) $f_{p}(x a y) \vee \alpha_{1} \geq f_{p}(x) \wedge f_{p}(y) \wedge \alpha_{2}$

(2) $f_{n}($ xay $) \wedge \beta_{2} \leq f_{n}(x) \vee f_{n}(y) \vee \beta_{1}$

for all $x, y, a \in S$.

Definition 9. $A$ BF set $f=\left(S ; f_{p}, f_{n}\right)$ on $S$ is called a $\left(\alpha_{1}, \alpha_{2} ; \beta_{1}, \beta_{2}\right)$-BF quasi-ideal on $S$, where $\alpha_{1}, \alpha_{2} \in[0,1]$, and $\beta_{1}, \beta_{2} \in[-1,0]$ if it satisfies the following conditions:

(1) $f_{p}(x) \vee \alpha_{1} \geq\left(f_{p} \bar{\circ} \mathcal{S}_{p}\right)(x) \wedge\left(\mathcal{S}_{p} \overline{0} f_{p}\right)(x) \wedge \alpha_{2}$

(2) $f_{n}(x) \wedge \beta_{2} \leq\left(f_{n} \circ \mathcal{S}_{n}\right)(x) \vee\left(\mathcal{S}_{n} \circ f_{n}\right)(x) \vee \beta_{1}$

for all $x, y \in S$.

In the following theorem, we give a relation between a bipolar $(\alpha, \beta)$-cut of $f$ and a $\left(\alpha_{1}, \alpha_{2} ; \beta_{1}, \beta_{2}\right)$-BF generalized bi-ideal on $S$.

Theorem 2. Let $f=\left(S ; f_{p}, f_{n}\right)$ be a BF set on a semigroup $S$ with $\operatorname{Im}\left(f_{p}\right) \subseteq \Delta^{+} \subseteq[0,1]$ and $\operatorname{Im}\left(f_{n}\right) \subseteq \Delta^{-} \subseteq[-1,0]$. Then $C(f ;(\alpha, \beta))(\neq \varnothing)$ is a generalized bi-ideal of $S$ for all $\alpha \in \Delta^{+}$and $\beta \in \Delta^{-}$if and only if $f$ is a $\left(\alpha_{1}, \alpha_{2} ; \beta_{1}, \beta_{2}\right)$-BF generalized bi-ideal on $S$ for all $\alpha_{1}, \alpha_{2} \in[0,1]$ and $\beta_{1}, \beta_{2} \in[-1,0]$.

Proof. Let $\alpha_{1}, \alpha_{2} \in[0,1], \beta_{1}, \beta_{2} \in[-1,0]$. Suppose on the contrary that $f$ is not a $\left(\alpha_{1}, \alpha_{2} ; \beta_{1}, \beta_{2}\right)$-BF generalized bi-ideal on $S$. Then there exists $x, y, a \in S$ such that

$$
f_{p}(x a y) \vee \alpha_{1}<f_{p}(x) \wedge f_{p}(y) \wedge \alpha_{2} \text { or } f_{n}(x a y) \wedge \beta_{2}>f_{n}(x) \vee f_{n}(y) \vee \beta_{1}
$$

Let $\alpha^{\prime}=f_{p}(x) \wedge f_{p}(y)$ and $\beta^{\prime}=f_{n}(x) \vee f_{n}(y)$. Then $x, y \in C\left(f ;\left(\alpha^{\prime}, \beta^{\prime}\right)\right)$. By assumption, we have xay $\in C\left(f ;\left(\alpha^{\prime}, \beta^{\prime}\right)\right)$. By Equation (1), $f_{p}(x a y) \leq f_{p}(x a y) \wedge \alpha_{1}<f_{p}(x) \wedge f_{p}(y) \wedge \alpha_{2} \leq f_{p}(x) \wedge f_{p}(y)=$ $\alpha^{\prime}$ or $f_{n}(x a y) \geq f_{n}(x a y) \wedge \beta_{2}>f_{n}(x) \vee f_{n}(y) \vee \beta_{1} \geq f_{n}(x) \vee f_{n}(y)=\beta^{\prime}$. Thus, xay $\notin C\left(f ;\left(\alpha^{\prime}, \beta^{\prime}\right)\right)$. This is a contradiction. Therefore $f$ is a $\left(\alpha_{1}, \alpha_{2} ; \beta_{1}, \beta_{2}\right)$-BF generalized bi-ideal on $S$.

Conversely, let $\alpha \in \Delta^{+}$, and $\beta \in \Delta^{-}$, and suppose that $C(f ;(\alpha, \beta)) \neq \varnothing$. Let $a \in S$ and $x, y \in C(f ;(\alpha, \beta))$. Then $f_{p}(x) \geq \alpha, f_{p}(y) \geq \alpha, f_{n}(x) \leq \beta$ and $f_{n}(y) \leq \beta$. By assumption, $f$ is a $\left(\alpha, f_{p}(x a y) ; f_{n}(x a y), \beta\right)$-BF generalized bi-ideal on $S$, and thus $f_{p}(x a y) \vee f_{p}(x a y) \geq f_{p}(x) \wedge f_{p}(y) \wedge \alpha$ and $f_{n}(x a y) \wedge f_{n}(x a y) \leq f_{n}(x) \vee f_{n}(y) \vee \beta$. Then $f_{p}(x a y) \geq f_{p}(x) \wedge f_{p}(y) \wedge \alpha \geq \alpha \wedge \alpha=\alpha$ and $f_{n}(x a y) \leq f_{n}(x) \vee f_{n}(x) \vee \beta \leq \beta \vee \beta=\beta$. Hence, xay $\in C(f ;(\alpha, \beta))$. Therefore $C(f ;(\alpha, \beta))$ is a generalized bi-ideal of $S$.

Corollary 1. Let $f=\left(S ; f_{n}, f_{p}\right)$ be a BF set on a semigroup. Then the following statements hold:

(1) $f$ is $a\left(\alpha_{1}, \alpha_{2} ; \beta_{1}, \beta_{2}\right)$-BF generalized bi-ideal on $S$ for all $\alpha_{1}, \alpha_{2} \in[0,1]$ and $\beta_{1}, \beta_{2} \in[-1,0]$ if and only if $C(f ;(\alpha, \beta))(\neq \varnothing)$ is a generalized bi-ideal of $S$ for all $\alpha \in \operatorname{Im}\left(f_{p}\right)$, and $\beta \in \operatorname{Im}\left(f_{n}\right)$;

(2) $f$ is a $\left(\alpha_{1}, \alpha_{2} ; \beta_{1}, \beta_{2}\right)$-BF generalized bi-ideal on $S$ for all $\alpha_{1}, \alpha_{2} \in[0,1]$, and $\beta_{1}, \beta_{2} \in[-1,0]$ if and only if $C(f ;(\alpha, \beta))(\neq \varnothing)$ is a generalized bi-ideal of $S$ for all $\alpha \in[0,1]$, and $\beta \in[-1,0]$.

Proof. (1) Set $\Delta^{+}=[0,1]$ and $\Delta^{-}=[-1,0]$, and apply Theorem 2.

(2) Set $\Delta^{+}=\operatorname{Im}\left(f_{p}\right)$ and $\Delta^{-}=\operatorname{Im}\left(f_{n}\right)$, and apply Theorem 2 .

Lemma 1. Every $\left(\alpha_{1}, \alpha_{2} ; \beta_{1}, \beta_{2}\right)$-BF generalized bi-ideal on a regular semigroup $S$ is a $\left(\alpha_{1}, \alpha_{2} ; \beta_{1}, \beta_{2}\right)$-BF bi-ideal on $S$. 
Proof. Let $S$ be a regular semigroup and $f=\left(S ; f_{p}, f_{n}\right)$ be a $\left(\alpha_{1}, \alpha_{2} ; \beta_{1}, \beta_{2}\right)$-BF generalized bi-ideal on $S$. Let $a, b \in S$; then there exists $x \in S$ such that $b=b x b$. Thus we have $f_{p}(a b) \vee \alpha_{1}=f_{p}(a(b x b)) \vee \alpha_{1}=f_{p}(a(b x) b) \vee \alpha_{1} \geq f_{p}(a) \wedge f_{p}(b) \wedge \alpha_{2}$ and $f_{n}(a b) \wedge \beta_{2}=f_{n}(a(b x b)) \wedge$ $\beta_{2}=f_{n}(a(b x) b) \wedge \beta_{2} \leq f_{n}(a) \vee f_{n}(b) \vee \beta_{1}$. This shows that $f$ is a $\left(\alpha_{1}, \alpha_{2} ; \beta_{1}, \beta_{2}\right)$-BF subsemigroup on $S$, and thus $f$ is a $\left(\alpha_{1}, \alpha_{2} ; \beta_{1}, \beta_{2}\right)$-BF bi-ideal on $S$.

Let $S$ be a semigroup and $\varnothing \neq I \subseteq S$. A positive characteristic function and a negative characteristic function are respectively defined by

$$
C_{I}^{p}: S \rightarrow[0,1], x \mapsto C_{I}^{p}(x):= \begin{cases}1, & x \in I \\ 0, & x \notin I\end{cases}
$$

and

$$
C_{I}^{n}: S \rightarrow[-1,0], x \mapsto C_{I}^{n}(x):= \begin{cases}-1, & x \in I \\ 0, & x \notin I\end{cases}
$$

\section{Remark 2.}

(1) For the sake of simplicity, we use the symbol $C_{I}=\left(S ; C_{I}^{p}, C_{I}^{n}\right)$ for the BF set. That is, $C_{I}=\left(S ; C_{I}^{p}, C_{I}^{n}\right)=\left(S ;\left(C_{I}\right)_{p},\left(C_{I}\right)_{n}\right)$. We call this a bipolar characteristic function.

(2) If I $=S$, then $C_{S}=\left(S ; C_{S}^{p}, C_{S}^{n}\right)$. In this case, we denote $\mathcal{S}=\left(S, \mathcal{S}_{p}, \mathcal{S}_{n}\right)$.

In the following theorem, some necessary and sufficient conditions of $\left(\alpha_{1}, \alpha_{2} ; \beta_{1}, \beta_{2}\right)$-BF generalized bi-ideals are obtained.

Theorem 3. Let $f=\left(S ; f_{p}, f_{n}\right)$ be a BF set on a semigroup $S$. Then the following statements are equivalent:

(1) $f$ is a $\left(\alpha_{1}, \alpha_{2} ; \beta_{1}, \beta_{2}\right)$-BF generalized bi-ideal on $S$.

(2) $f_{p} \stackrel{\left(\alpha_{2}, \alpha_{1}\right)}{\circ} \mathcal{S}_{p} \stackrel{\left(\alpha_{2}, \alpha_{1}\right)}{\circ} f_{p} \leq f_{p}^{\left(\alpha_{2}, \alpha_{1}\right)}$ and $f_{n} \underset{\left(\beta_{2}, \beta_{1}\right)}{\circ} \mathcal{S}_{n} \underset{\left(\beta_{2}, \beta_{1}\right)}{\circ} f_{n} \geq f_{n}^{\left(\beta_{2}, \beta_{1}\right)}$.

Proof. $(\Rightarrow)$ Let $a$ be any element of $S$. In the case for which $\left(f_{p} \stackrel{\left(\alpha_{2}, \alpha_{1}\right)}{\circ} \mathcal{S}_{p} \stackrel{\left(\alpha_{2}, \alpha_{1}\right)}{o} f_{p}\right)(a)=0$, it is clear that $f_{p} \stackrel{\left(\alpha_{2}, \alpha_{1}\right)}{\circ} \mathcal{S}_{p} \stackrel{\left(\alpha_{2}, \alpha_{1}\right)}{\circ} f_{p} \leq f_{p}^{\left(\alpha_{2}, \alpha_{1}\right)}$. Otherwise, there exist $x, y, r, s \in S$ such that $a=x y$ and $x=r s$. Because $f$ is a $\left(\alpha_{1}, \alpha_{2} ; \beta_{1}, \beta_{2}\right)$-BF generalized bi-ideal on $S$, we have $f_{p}(r s y) \vee \alpha_{1} \geq f_{p}(r) \wedge f_{p}(s) \wedge \alpha_{2}$ and $f_{n}(r s y) \wedge \beta_{2} \leq f_{n}(r) \vee f_{n}(s) \vee \beta_{1}$. Consider

$$
\begin{aligned}
\left(f_{p} \underset{\substack{\left(\alpha_{2}, \alpha_{1}\right) \\
\mathcal{S}_{p}}}{\left(\alpha_{2}, \alpha_{1}\right)} f_{p}\right)(a) & =\left(\left(f_{p} \overline{0} \mathcal{S}_{p} \overline{0} f_{p}\right)(a) \wedge \alpha_{2}\right) \vee \alpha_{1} \\
& =\left(\bigvee_{a=x y}\left\{\left(f_{p} \overline{0} \mathcal{S}_{p}\right)(x) \wedge f_{p}(y)\right\} \wedge \alpha_{2}\right) \vee \alpha_{1} \\
& =\left(\bigvee_{a=x y}\left\{\bigvee_{x=r s}\left\{f_{p}(r) \wedge \mathcal{S}_{p}(s)\right\} \wedge f_{p}(y)\right\} \wedge \alpha_{2}\right) \vee \alpha_{1} \\
& =\left(\bigvee_{a=x y}\left\{\bigvee_{x=r s}\left\{f_{p}(r) \wedge 1\right\} \wedge f_{p}(y)\right\} \wedge \alpha_{2}\right) \vee \alpha_{1} \\
& =\left(\bigvee_{a=r s y}\left\{f_{p}(r) \wedge f_{p}(y) \wedge \alpha_{2}\right\} \wedge \alpha_{2}\right) \vee \alpha_{1} \\
& \leq\left(\bigvee_{a=r s y}\left\{f_{p}(r s y) \vee \alpha_{1}\right\} \wedge \alpha_{2}\right) \vee \alpha_{1} \\
& \leq\left(\bigvee_{a=r s y}\left\{f_{p}(a) \vee \alpha_{1}\right\} \wedge \alpha_{2}\right) \vee \alpha_{1} \\
& \leq\left(\left(f_{p}(a) \vee \alpha_{1}\right) \wedge \alpha_{2}\right) \vee \alpha_{1} \\
& =\left(f_{p}(a) \wedge \alpha_{2}\right) \vee \alpha_{1} \\
& =f_{p}^{\left(\alpha_{2}, \alpha_{1}\right)}(a)
\end{aligned}
$$


Hence $f_{p} \stackrel{\left(\alpha_{2}, \alpha_{1}\right)}{\circ} \mathcal{S}_{p} \stackrel{\left(\alpha_{2}, \alpha_{1}\right)}{\circ} f_{p} \leq f_{p}^{\left(\alpha_{2}, \alpha_{1}\right)}$.

Similarly, we can show that $f_{n} \underset{\left(\beta_{2}, \beta_{1}\right)}{\circ} \mathcal{S}_{n} \underset{\left(\beta_{2}, \beta_{1}\right)}{\circ} f_{n} \geq f_{n}^{\left(\beta_{2}, \beta_{1}\right)}$.

$(\Leftarrow)$ Conversely, let $a, x, y, z \in S$ such that $a=x y z$. Then we have

$$
\begin{aligned}
f_{p}(x y z) \vee \alpha_{1} & \geq\left(f_{p}(a) \wedge \alpha_{2}\right) \vee \alpha_{1} \\
& =f_{p}^{\left(\alpha_{2}, \alpha_{1}\right)}(a) \\
& \geq\left(f_{p} \stackrel{\left(\alpha_{2}, \alpha_{1}\right)}{\circ} \mathcal{S}_{p} \stackrel{\left(\alpha_{2}, \alpha_{1}\right)}{\circ} f_{p}\right)(a) \\
& =\left(\left(f_{p} \bar{\circ} \mathcal{S}_{p} \bar{\circ} f_{p}\right)(a) \wedge \alpha_{2}\right) \vee \alpha_{1} \\
& =\left(\bigvee_{a=b c}\left\{\left(f_{p} \bar{\circ} \mathcal{S}_{p}\right)(b) \wedge f_{p}(c)\right\} \wedge \alpha_{2}\right) \vee \alpha_{1} \\
& \geq\left(\left(f_{p} \bar{\circ} \mathcal{S}_{p}\right)(x y) \wedge f_{p}(z) \wedge \alpha_{2}\right) \vee \alpha_{1} \\
& =\left(\bigvee_{x y=r s}\left\{f_{p}(r) \wedge \mathcal{S}_{p}(s)\right\} \wedge f_{p}(z) \wedge \alpha_{2}\right) \vee \alpha_{1} \\
& \geq\left(f_{p}(x) \wedge \mathcal{S}_{p}(y) \wedge f_{p}(z) \wedge \alpha_{2}\right) \vee \alpha_{1} \\
\geq & \left(f_{p}(x) \wedge f_{p}(z) \wedge \alpha_{2}\right) \vee \alpha_{1} \\
\geq & f_{p}(x) \wedge f_{p}(z) \wedge \alpha_{2}
\end{aligned}
$$

Similarly, we can show that $f_{n}(x y z) \wedge \beta_{2} \leq f_{n}(x) \vee f_{n}(z) \vee \beta_{1}$ for all $x, y, z \in S$. Therefore $f$ is a $\left(\alpha_{1}, \alpha_{2} ; \beta_{1}, \beta_{2}\right)$-BF generalized bi-ideal on $S$ for all $\alpha_{1}, \alpha_{2} \in[0,1]$ and $\beta_{1}, \beta_{2} \in[-1,0]$.

Theorem 4. Let $f=\left(S ; f_{p}, f_{n}\right)$ be a BF set on a semigroup $S$. Then the following statements are equivalent:

(1) $f$ is a $\left(\alpha_{1}, \alpha_{2} ; \beta_{1}, \beta_{2}\right)$-BF bi-ideal on $S$.

(2) $f_{p} \stackrel{\left(\alpha_{2}, \alpha_{1}\right)}{\circ} \mathcal{S}_{p} \stackrel{\left(\alpha_{2}, \alpha_{1}\right)}{\circ} f_{p} \leq f_{p}^{\left(\alpha_{2}, \alpha_{1}\right)}$ and $f_{n} \underset{\left(\beta_{2}, \beta_{1}\right)}{\circ} \mathcal{S}_{n} \underset{\left(\beta_{2}, \beta_{1}\right)}{\circ} f_{n} \geq f_{n}^{\left(\beta_{2}, \beta_{1}\right)}$.

Proof. The proof is similar to the proof of Theorem 3.

In the following theorem, we give a relation between a bipolar $(\alpha, \beta)$-cut of $f$ and a $\left(\alpha_{1}, \alpha_{2} ; \beta_{1}, \beta_{2}\right)$-BF quasi-ideal on $S$.

Theorem 5. Let $f=\left(S ; f_{p}, f_{n}\right)$ be a BF set on a semigroup $S$ with $\operatorname{Im}\left(f_{p}\right) \subseteq \Delta^{+} \subseteq[0,1]$ and $\operatorname{Im}\left(f_{n}\right) \subseteq \Delta^{-} \subseteq[-1,0]$. Then $C(f ;(\alpha, \beta))(\neq \varnothing)$ is a quasi-ideal of $S$ for all $\alpha \in \Delta^{+}$and $\beta \in \Delta^{-}$if and only if $f$ is $a\left(\alpha_{1}, \alpha_{2} ; \beta_{1}, \beta_{2}\right)$-BF quasi-ideal on $S$ for all $\alpha_{1}, \alpha_{2} \in[0,1]$ and $\beta_{1}, \beta_{2} \in[-1,0]$.

Proof. $(\Rightarrow)$ Let $\alpha_{1}, \alpha_{2} \in[0,1]$ and $\beta_{1}, \beta_{2} \in[-1,0]$. Suppose on the contrary that $f$ is not a $\left(\alpha_{1}, \alpha_{2} ; \beta_{1}, \beta_{2}\right)$-BF quasi-ideal on $S$. Then there exists $x \in S$ such that

$$
f_{p}(x) \vee \alpha_{1}<\left(f_{p} \bar{\circ} \mathcal{S}_{p}\right)(x) \wedge\left(\mathcal{S}_{p} \bar{\sigma} f_{p}\right)(x) \wedge \alpha_{2}
$$

or

$$
f_{n}(x) \wedge \beta_{2}>\left(f_{n} \circ \mathcal{S}_{n}\right)(x) \vee\left(\mathcal{S}_{n} \circ f_{n}\right)(x) \vee \beta_{1}
$$

Case 1: $f_{p}(x) \vee \alpha_{1}<\left(f_{p} \bar{\circ} \mathcal{S}_{p}\right)(x) \wedge\left(\mathcal{S}_{p} \bar{\circ} f_{p}\right)(x) \wedge \alpha_{2}$. Let $\alpha^{\prime}=\left(f_{p} \bar{\circ} \mathcal{S}_{p}\right)(x) \wedge\left(\mathcal{S}_{p} \bar{\circ} f_{p}\right)(x)$. Then $\alpha^{\prime} \leq\left(f_{p} \bar{\circ} \mathcal{S}_{p}\right)(x), \alpha^{\prime} \leq\left(\mathcal{S}_{p} \bar{\circ} f_{p}\right)(x)$. This implies that there exist $a, b, c, d \in S$ such that $x=a b=c d$. Then

$$
\alpha^{\prime} \leq\left(f_{p} \bar{\circ} \mathcal{S}_{p}\right)(x)=\bigvee_{x=y z}\left\{f_{p}(y) \wedge \mathcal{S}_{p}(z)\right\} \leq f_{p}(a) \wedge \mathcal{S}_{p}(b)=f_{p}(a)
$$




$$
\alpha^{\prime} \leq\left(\mathcal{S}_{p} \bar{\sigma} f_{p}\right)(x)=\bigvee_{x=y z}\left\{\mathcal{S}_{p}(z) \wedge f_{p}(y)\right\} \leq \mathcal{S}_{p}(c) \wedge f_{p}(d)=f_{p}(d)
$$

Let $\beta^{\prime}=f_{n}(a) \vee f_{n}(d)$. Then $f_{n}(a) \leq \beta^{\prime}$ and $f_{n}(d) \leq \beta^{\prime}$.

Thus $a, d \in C\left(f ;\left(\alpha^{\prime}, \beta^{\prime}\right)\right)$, and so $a d \in C\left(f ;\left(\alpha^{\prime}, \beta^{\prime}\right)\right) S$ and $a d \in S C\left(f ;\left(\alpha^{\prime}, \beta^{\prime}\right)\right)$. Hence $x \in C\left(f ;\left(\alpha^{\prime}, \beta^{\prime}\right)\right) S$ and $x \in S C\left(f ;\left(\alpha^{\prime}, \beta^{\prime}\right)\right)$, and it follows that $x \in C\left(f ;\left(\alpha^{\prime}, \beta^{\prime}\right)\right) S \cap S C\left(f ;\left(\alpha^{\prime}, \beta^{\prime}\right)\right)$. By hypothesis, $x \in C\left(f ;\left(\alpha^{\prime}, \beta^{\prime}\right)\right)$.

Case 2: $f_{n}(x) \wedge \beta_{2}>\left(f_{n} \circ \mathcal{S}_{n}\right)(x) \vee\left(\mathcal{S}_{n} \circ f_{n}\right)(x) \vee \beta_{1}$. Let $\beta^{\prime}=\left(f_{n} \circ \mathcal{S}_{n}\right)(x) \vee\left(\mathcal{S}_{n} \circ f_{n}\right)(x)$. Then $\beta^{\prime} \geq\left(f_{n} \circ \mathcal{S}_{n}\right)(x)$ and $\beta^{\prime} \geq\left(\mathcal{S}_{n} \circ f_{n}\right)(x)$. This implies that there exist $a^{\prime}, b^{\prime}, c^{\prime}, d^{\prime} \in S$ such that $x=a^{\prime} b^{\prime}=c^{\prime} d^{\prime}$. Then

$$
\begin{aligned}
& \beta^{\prime} \geq\left(f_{n} \circ \mathcal{S}_{n}\right)(x)=\bigwedge_{x=y z}\left\{f_{n}(y) \vee \mathcal{S}_{n}(z)\right\} \geq f_{n}\left(a^{\prime}\right) \vee \mathcal{S}_{n}\left(b^{\prime}\right) \geq f_{n}\left(a^{\prime}\right) \\
& \beta^{\prime} \geq\left(\mathcal{S}_{n} \circ f_{n}\right)(x)=\bigwedge_{x=y z}\left\{\mathcal{S}_{n}(z) \vee f_{n}(y)\right\} \geq \mathcal{S}_{n}\left(c^{\prime}\right) \vee f_{n}\left(d^{\prime}\right) \geq f_{n}\left(d^{\prime}\right)
\end{aligned}
$$

Let $\alpha^{\prime}=f_{p}\left(a^{\prime}\right) \wedge f_{p}\left(d^{\prime}\right)$. Then $f_{p}\left(a^{\prime}\right) \geq \alpha^{\prime}$ and $f_{p}\left(d^{\prime}\right) \geq \alpha^{\prime}$. Thus $a^{\prime}, d^{\prime} \in C\left(f ;\left(\alpha^{\prime}, \beta^{\prime}\right)\right)$, and so $a^{\prime} d^{\prime} \in C\left(f ;\left(\alpha^{\prime}, \beta^{\prime}\right)\right) S$ and $a^{\prime} d^{\prime} \in S C\left(f ;\left(\alpha^{\prime}, \beta^{\prime}\right)\right)$. Hence $x \in C\left(f ;\left(\alpha^{\prime}, \beta^{\prime}\right)\right) S$ and $x \in S C\left(f ;\left(\alpha^{\prime}, \beta^{\prime}\right)\right)$, and it follows that $x \in C\left(f ;\left(\alpha^{\prime}, \beta^{\prime}\right)\right) S \cap S C\left(f ;\left(\alpha^{\prime}, \beta^{\prime}\right)\right)$. By hypothesis, $x \in C\left(f ;\left(\alpha^{\prime}, \beta^{\prime}\right)\right)$. Therefore $x \in C\left(f ;\left(\alpha^{\prime}, \beta^{\prime}\right)\right)$. By Equation (2),

$$
f_{p}(x) \leq f_{p}(x) \vee \alpha_{1}<\left(f_{p} \bar{\circ} \mathcal{S}_{p}\right)(x) \wedge\left(\mathcal{S}_{p} \overline{0} f_{p}\right)(x) \wedge \alpha_{2} \leq\left(f_{p} \bar{\circ} \mathcal{S}_{p}\right)(x) \wedge\left(\mathcal{S}_{p} \bar{\sigma} f_{p}\right)(x)=\alpha^{\prime}
$$

or

$$
f_{n}(x) \geq f_{n}(x) \wedge \beta_{2}>\left(f_{n} \circ \mathcal{S}_{n}\right)(x) \vee\left(\mathcal{S}_{n} \circ f_{n}\right)(x) \vee \beta_{1} \geq\left(f_{n} \circ \mathcal{S}_{n}\right)(x) \vee\left(\mathcal{S}_{n} \circ f_{n}\right)(x)=\beta^{\prime}
$$

and it follows that $x \notin C\left(f ;\left(\alpha^{\prime}, \beta^{\prime}\right)\right)$. This is a contradiction. Therefore $f$ is a $\left(\alpha_{1}, \alpha_{2} ; \beta_{1}, \beta_{2}\right)$-BF quasi-ideal on $S$.

$(\Leftarrow)$ Conversely, let $\alpha \in \Delta^{+}$and $\beta \in \Delta^{-}$, and suppose that $C(f ;(\alpha, \beta)) \neq \varnothing$. Let $x \in S$ be such that $x \in C(f ;(\alpha, \beta)) S \cap S C(f ;(\alpha, \beta))$. Then $x \in C(f ;(\alpha, \beta)) S$ and $x \in S C(f ;(\alpha, \beta))$. Thus there exist $y, z^{\prime} \in C(f ;(\alpha, \beta))$ and $z, y^{\prime} \in S$ such that $x=y z$ and $x=y^{\prime} z^{\prime}$.

By assumption, $f$ is a $\left(f_{p}(x), \alpha ; \beta, f_{n}(x)\right)$-BF quasi-ideal on $S$, and thus

$$
\begin{aligned}
f_{p}(x) & =f_{p}(x) \vee f_{p}(x) \\
& \geq\left(f_{p} \bar{\circ} \mathcal{S}_{p}\right)(x) \wedge\left(\mathcal{S}_{p} \overline{0} f_{p}\right)(x) \wedge \alpha \\
& =\bigvee_{x=y z}\left\{f_{p}(y) \wedge \mathcal{S}_{p}(z)\right\} \wedge \bigvee_{x=y^{\prime} z^{\prime}}\left\{\mathcal{S}_{p}\left(y^{\prime}\right) \wedge f_{p}\left(z^{\prime}\right)\right\} \wedge \alpha \\
& =\bigvee_{x=y z}\left\{f_{p}(y) \wedge 1\right\} \wedge \bigvee_{x=y^{\prime} z^{\prime}}\left\{1 \wedge f_{p}\left(z^{\prime}\right)\right\} \wedge \alpha \\
& =\bigvee_{x=y z}\left\{f_{p}(y)\right\} \wedge \bigvee_{x=y^{\prime} z^{\prime}}\left\{f_{p}\left(z^{\prime}\right)\right\} \wedge \alpha \\
& \geq f_{p}(y) \wedge f_{p}\left(z^{\prime}\right) \wedge \alpha
\end{aligned}
$$

Because $y, z^{\prime} \in C(f ;(\alpha, \beta))$, we have $f_{p}(y) \geq \alpha$ and $f_{p}\left(z^{\prime}\right) \geq \alpha$. Then $f_{p}(x) \geq \alpha$. Similarly, we can show that $f_{n}(x) \leq \beta$. Hence, $x \in C(f ;(\alpha, \beta))$. Therefore $C(f ;(\alpha, \beta))$ is a quasi-ideal of $S$.

Corollary 2. Let $f=\left(S ; f_{n}, f_{p}\right)$ be a BF set on a semigroup $S$. Then

(1) $f$ is a $\left(\alpha_{1}, \alpha_{2} ; \beta_{1}, \beta_{2}\right)$-BF quasi-ideal on $S$ for all $\alpha_{1}, \alpha_{2} \in[0,1]$ and $\beta_{1}, \beta_{2} \in[-1,0]$ if and only if $C(f ;(\alpha, \beta))(\neq \varnothing)$ is a quasi-ideal of $S$ for all $\alpha \in \operatorname{Im}\left(f_{p}\right)$ and $\beta \in \operatorname{Im}\left(f_{n}\right)$;

(2) $f$ is $a\left(\alpha_{1}, \alpha_{2} ; \beta_{1}, \beta_{2}\right)$-BF quasi-ideal on $S$ for all $\alpha_{1}, \alpha_{2} \in[0,1]$ and $\beta_{1}, \beta_{2} \in[-1,0]$ if and only if $C(f ;(\alpha, \beta))(\neq \varnothing)$ is a quasi-ideal of $S$ for all $\alpha \in[0,1]$ and $\beta \in[-1,0]$. 
Proof. (1) Set $\Delta^{+}=[0,1]$ and $\Delta^{-}=[-1,0]$, and apply Theorem 5 .

(2) Set $\Delta^{+}=\operatorname{Im}\left(f_{p}\right)$ and $\Delta^{-}=\operatorname{Im}\left(f_{n}\right)$, and apply Theorem 5 .

In the following theorem, we discuss a quasi-ideal of a semigroup $S$ in terms of the bipolar characteristic function being a $\left(\alpha_{1}, \alpha_{2} ; \beta_{1}, \beta_{2}\right)$-BF quasi-ideal on $S$.

Theorem 6. Let $S$ be a semigroup. Then a non-empty subset $I$ is a quasi-ideal of $S$ if and only if the bipolar characteristic function $C_{I}=\left(S ; C_{I}^{p}, C_{I}^{n}\right)$ is a $\left(\alpha_{1}, \alpha_{2} ; \beta_{1}, \beta_{2}\right)$-BF quasi-ideal on $S$ for all $\alpha_{1}, \alpha_{2} \in[0,1]$ and $\beta_{1}, \beta_{2} \in[-1,0]$.

Proof. $(\Rightarrow)$ Let $I$ be a quasi-ideal of $S$ and $x \in S$. Let $\alpha_{1}, \alpha_{2} \in[0,1]$ and $\beta_{1}, \beta_{2} \in[-1,0]$.

Case 1: $x, y \in I$. Then

$$
\begin{gathered}
C_{I}^{p}(x) \vee \alpha_{1}=1 \geq\left(C_{I}^{p} \overline{\bar{o}} \mathcal{S}_{p}\right)(x) \wedge\left(\mathcal{S}_{p} \bar{\circ} C_{I}^{p}\right)(x) \wedge \alpha_{2} \\
C_{I}^{n}(x) \wedge \beta_{2}=-1 \leq\left(C_{I}^{n} \circ \mathcal{S}_{n}\right)(x) \vee\left(\mathcal{S}_{n} \circ C_{I}^{n}\right)(x) \vee \beta_{1}
\end{gathered}
$$

Case 2: $x \notin I$. Then $x \notin S I$ or $x \notin I S$. If $x \notin S I$, then $\left(C_{I}^{p} \overline{\mathcal{S}_{p}}\right)(x)=0$ and $\left(C_{I}^{n} \circ \mathcal{S}_{n}\right)(x)=0$. Thus

$$
\begin{aligned}
& C_{I}^{p}(x) \vee \alpha_{1} \geq 0=\left(C_{I}^{p} \bar{\circ} \mathcal{S}_{p}\right)(x) \wedge\left(\mathcal{S}_{p} \bar{\circ} C_{I}^{p}\right)(x) \wedge \alpha_{2} \\
& C_{I}^{n}(x) \wedge \beta_{2} \leq 0=\left(C_{I}^{n} \circ \mathcal{S}_{n}\right)(x) \vee\left(\mathcal{S}_{n} \circ C_{I}^{n}\right)(x) \vee \beta_{1}
\end{aligned}
$$

Therefore $C_{I}=\left(S ; C_{I}^{p}, C_{I}^{n}\right)$ is a $\left(\alpha_{1}, \alpha_{2} ; \beta_{1}, \beta_{2}\right)$-BF quasi-ideal on $S$.

$(\Leftarrow)$ Conversely, let $C_{I}$ be a $\left(\alpha_{1}, \alpha_{2} ; \beta_{1}, \beta_{2}\right)$-BF quasi-ideal on $S$ for all $\alpha_{1}, \alpha_{2} \in[0,1]$ and $\beta_{1}, \beta_{2} \in[-1,0]$. Let $a \in I S \cap S I$. Then there exist $a, b \in S$ and $x, y \in I$ such that $a=x b=c y$. Then $\left(C_{I}\right)_{p}(x)=1=\left(C_{I}\right)_{p}(y)$ and $\left(C_{I}\right)_{n}(x)=-1=\left(C_{I}\right)_{n}(y)$. Hence $x, y \in C\left(C_{I} ;(1,-1)\right)$, and so $x b \in C\left(C_{I} ;(1,-1)\right) S$ and $c y \in S C\left(C_{I} ;(1,-1)\right)$. Hence $a \in C\left(C_{I} ;(1,-1)\right) S$ and $a \in S C\left(C_{I} ;(1,-1)\right)$, and it follows that $a \in C\left(C_{I} ;(1,-1)\right) S \cap S C\left(C_{I} ;(1,-1)\right)$. By Corollary $2, C\left(C_{I} ;(1,-1)\right)$ is a quasi-ideal. Thus $a \in C\left(C_{I} ;(1,-1)\right)$, and so $C_{I}^{p}(a) \geq 1$. This implies that $a \in I$. Therefore $I$ is a quasi-ideal on $S$.

Theorem 7. Let $S$ be a semigroup. Then I is a generalized bi-ideal of $S$ if and only if the bipolar characteristic function $C_{I}=\left(S ; C_{I}^{p}, C_{I}^{n}\right)$ is a $\left(\alpha_{1}, \alpha_{2} ; \beta_{1}, \beta_{2}\right)$-BF generalized bi-ideal on $S$ for all $\alpha_{1}, \alpha_{2} \in[0,1]$ and $\beta_{1}, \beta_{2} \in[-1,0]$.

Proof. $(\Rightarrow)$ Let $I$ be a generalized bi-ideal of $S$ and $x, y, a \in S$. Let $\alpha_{1}, \alpha_{2} \in[0,1]$ and $\beta_{1}, \beta_{2} \in[-1,0]$. Case 1: $x, y \in I$. Then $x a y \in I$; thus

$$
C_{I}^{p}(x a y) \vee \alpha_{1}=1 \geq C_{I}^{p}(x) \wedge C_{I}^{p}(y) \wedge \alpha_{2}
$$

and

$$
C_{I}^{n}(x a y) \wedge \beta_{2}=-1 \leq C_{I}^{n}(x) \vee C_{I}^{n}(y) \vee \beta_{1}
$$

Case 2: $x \notin I$ or $y \notin I$. Then

$$
C_{I}^{p}(x a y) \vee \alpha_{1} \geq 0=C_{I}^{p}(x) \wedge C_{I}^{p}(y) \wedge \alpha_{2}
$$

and

$$
C_{I}^{n}(x a y) \wedge \beta_{2} \leq 0=C_{I}^{n}(x) \vee C_{I}^{n}(y) \vee \beta_{1}
$$

Therefore $C_{I}=\left(S ; C_{I}^{p}, C_{I}^{n}\right)$ is a $\left(\alpha_{1}, \alpha_{2} ; \beta_{1}, \beta_{2}\right)$-BF generalized bi-ideal on $S$.

$(\Leftarrow)$ Conversely, let $C_{I}$ be a $\left(\alpha_{1}, \alpha_{2} ; \beta_{1}, \beta_{2}\right)$-BF generalized bi-ideal on $S$ for all $\alpha_{1}, \alpha_{2} \in[0,1]$ and $\beta_{1}, \beta_{2} \in[-1,0]$. Let $a \in S$ and $x, y \in I$. Then $\left(C_{I}\right)_{p}(x)=1=\left(C_{I}\right)_{p}(y)$ and $\left(C_{I}\right)_{n}(x)=-1=\left(C_{I}\right)_{n}(y)$. Hence, $x, y \in C\left(C_{I} ;(1,-1)\right)$. By Corollary $1, C\left(C_{I} ;(1,-1)\right)$ is a generalized bi-ideal. Thus xay $\in C\left(C_{I} ;(1,-1)\right)$, and so $C_{I}^{p}($ xay $) \geq 1$. This implies that xay $\in I$. Therefore $I$ is a generalized bi-ideal on $S$. 
Theorem 8. Every $\left(\alpha_{1}, \alpha_{2} ; \beta_{1}, \beta_{2}\right)$-BF left (right) ideal on a semigroup $S$ is a $\left(\alpha_{1}, \alpha_{2} ; \beta_{1}, \beta_{2}\right)$-BF quasi-ideal on $S$.

Proof. Let $f=\left(S ; f_{p}, f_{n}\right)$ be a $\left(\alpha_{1}, \alpha_{2} ; \beta_{1}, \beta_{2}\right)$-BF left ideal on $S$ and $x, y \in S$. Then

$$
\begin{aligned}
(\mathcal{S} \bar{\circ} f)_{p}(x y) \wedge \alpha_{2} & =\left(\mathcal{S}_{p} \bar{\circ} f_{p}\right)(x y) \wedge \alpha_{2} \\
& =\left(\bigvee_{x=y z}\left\{\mathcal{S}_{p}(y) \wedge f_{p}(z)\right\}\right) \wedge \alpha_{2} \\
& =\bigvee_{x=y z}\left\{f_{p}(z)\right\} \wedge \alpha_{2} \\
& \leq \bigvee_{x=y z}\left\{f_{p}(y z)\right\} \vee \alpha_{1} \\
& \leq f_{p}(x) \vee \alpha_{1}
\end{aligned}
$$

Thus $f_{p}(x) \vee \alpha_{1} \geq\left(\mathcal{S}_{p} \bar{\circ} f_{p}\right)(x y) \wedge \alpha_{2}$. Hence $f_{p}(x) \vee \alpha_{1} \geq\left(\mathcal{S}_{p} \bar{\circ} f_{p}\right)(x y) \wedge \alpha_{2} \geq\left(\mathcal{S}_{p} \bar{\circ} f_{p}\right)(x y) \wedge$ $\left(f_{p} \bar{\circ} \mathcal{S}_{p}\right)(x y) \wedge \alpha_{2}$. Similarly, we can show that $f_{n}(x) \wedge \beta_{2} \leq\left(f_{n} \circ \mathcal{S}_{n}\right)(x) \vee\left(\mathcal{S}_{n} \circ f_{n}\right)(x) \vee \beta_{1}$. Therefore $f$ is a $\left(\alpha_{1}, \alpha_{2} ; \beta_{1}, \beta_{2}\right)$-BF quasi-ideal on $S$.

Lemma 2. Every $\left(\alpha_{1}, \alpha_{2} ; \beta_{1}, \beta_{2}\right)$-BF quasi-ideal on a semigroup $S$ is a $\left(\alpha_{1}, \alpha_{2} ; \beta_{1}, \beta_{2}\right)$-BF bi-ideal on $S$.

Proof. Let $f=\left(S ; f_{p}, f_{n}\right)$ be a $\left(\alpha_{1}, \alpha_{2} ; \beta_{1}, \beta_{2}\right)$-BF quasi-ideal on $S$ and $x, y, z \in S$. Then

$$
\begin{aligned}
f_{p}(x y) \vee \alpha_{1} & \geq\left(f_{p} \bar{\circ} \mathcal{S}_{p}\right)(x y) \wedge\left(\mathcal{S}_{p} \bar{\circ} f_{p}\right)(x y) \wedge \alpha_{2} \\
& =\bigvee_{x y=a b}\left\{f_{p}(a) \wedge \mathcal{S}_{p}(b)\right\} \wedge \bigvee_{x y=r s}\left\{\mathcal{S}_{p}(r) \wedge f_{p}(s)\right\} \wedge \alpha_{2} \\
& \geq f_{p}(x) \wedge \mathcal{S}_{p}(y) \wedge \mathcal{S}_{p}(x) \wedge f_{p}(y) \wedge \alpha_{2} \\
& \geq f_{p}(x) \wedge 1 \wedge 1 \wedge f_{p}(y) \wedge \alpha_{2} \\
& =f_{p}(x) \wedge f_{p}(y) \wedge \alpha_{2}
\end{aligned}
$$

Hence, $f_{p}(x y) \vee \alpha_{1} \geq f_{p}(x) \wedge f_{p}(y) \wedge \alpha_{2}$. Additionally,

$$
\begin{aligned}
f_{p}(x y z) \vee \alpha_{1} & \geq\left(f_{p} \bar{\circ} \mathcal{S}_{p}\right)(x y z) \wedge\left(\mathcal{S}_{p} \bar{\circ} f_{p}\right)(x y z) \wedge \alpha_{2} \\
& =\bigvee_{x y z=a b}\left\{f_{p}(a) \wedge \mathcal{S}_{p}(b)\right\} \wedge \bigvee_{x y z=r s}\left\{\mathcal{S}_{p}(r) \wedge f_{p}(s)\right\} \wedge \alpha_{2} \\
& \geq f_{p}(x) \wedge \mathcal{S}_{p}(y z) \wedge \mathcal{S}_{p}(x y) \wedge f_{p}(z) \wedge \alpha_{2} \\
& \geq f_{p}(x) \wedge 1 \wedge 1 \wedge f_{p}(z) \wedge \alpha_{2} \\
& =f_{p}(x) \wedge f_{p}(z) \wedge \alpha_{2}
\end{aligned}
$$

Hence, $f_{p}(x y z) \vee \alpha_{1} \geq f_{p}(x) \wedge f_{p}(z) \wedge \alpha_{2}$. Similarly, we can show that $f_{n}(x y) \wedge \beta_{2} \leq f_{n}(x) \vee$ $f_{n}(y) \vee \beta_{1}$ and $f_{n}(x y z) \wedge \beta_{2} \leq f_{n}(x) \vee f_{n}(z) \vee \beta_{1}$. Therefore $f$ is a $\left(\alpha_{1}, \alpha_{2} ; \beta_{1}, \beta_{2}\right)$-BF bi-ideal on $S$.

Lemma 3. Let $A$ and $B$ be non-empty subsets of a semigroup S. Then the following conditions hold:

(1) $\quad\left(C_{A}\right)_{p} \stackrel{\left(\alpha_{2}, \alpha_{1}\right)}{\wedge}\left(C_{B}\right)_{p}=\left(C_{A \cap B}\right)_{p}^{\left(\alpha_{2}, \alpha_{1}\right)}$.

(2) $\quad\left(C_{A}\right)_{n} \stackrel{\left(\beta_{2}, \beta_{1}\right)}{V}\left(C_{B}\right)_{n}=\left(C_{A \cup B}\right)_{n}^{\left(\beta_{2}, \beta_{1}\right)}$.

(3) $\left(C_{A}\right)_{p} \stackrel{\left(\alpha_{2}, \alpha_{1}\right)}{\circ}\left(C_{B}\right)_{p}=\left(C_{A B}\right)_{p}^{\left(\alpha_{2}, \alpha_{1}\right)}$.

(4) $\quad\left(C_{A}\right)_{n} \stackrel{\left(\beta_{2}, \beta_{1}\right)}{\circ}\left(C_{B}\right)_{n}=\left(C_{A B}\right)_{n}^{\left(\beta_{2}, \beta_{1}\right)}$. 
Lemma 4. If $f=\left(S ; f_{p}, f_{n}\right)$ is a $\left(\alpha_{1}, \alpha_{2} ; \beta_{1}, \beta_{2}\right)$-BF left ideal and $g=\left(S ; g_{p}, g_{n}\right)$ is $a\left(\alpha_{1}, \alpha_{2} ; \beta_{1}, \beta_{2}\right)$-BF right ideal on a semigroup $S$, then $f_{p} \stackrel{\left(\alpha_{2}, \alpha_{1}\right)}{\circ} g_{p} \leq f_{p} \stackrel{\left(\alpha_{2}, \alpha_{1}\right)}{\wedge} g_{p}$ and $f_{n} \underset{\left(\beta_{2}, \beta_{1}\right)}{\circ} g_{n} \geq f_{\left(\beta_{2}, \beta_{1}\right)} \vee g_{n}$.

Theorem 9. For a semigroup $S$, the following are equivalent.

(1) $S$ is regular.

(2) $f_{p} \stackrel{\left(\alpha_{2}, \alpha_{1}\right)}{\wedge} g_{p}=f_{p} \stackrel{\left(\alpha_{2}, \alpha_{1}\right)}{\circ} g_{p}$ and $f_{n} \underset{\left(\beta_{2}, \beta_{1}\right)}{\vee} g_{n}=f_{n} \underset{\left(\beta_{2}, \beta_{1}\right)}{\circ} g_{n}$ for every $\left(\alpha_{1}, \alpha_{2} ; \beta_{1}, \beta_{2}\right)$-BF right ideal $f=\left(S ; f_{p}, f_{n}\right)$ and every $\left(\alpha_{1}, \alpha_{2} ; \beta_{1}, \beta_{2}\right)$-BF left ideal $g=\left(S ; g_{p}, g_{n}\right)$ on $S$.

Next, we characterize a regular semigroup by generalizations of BF subsemigroups.

Theorem 10. For a semigroup $S$, the following are equivalent.

(1) $S$ is regular.

(2) $f_{p} \stackrel{\left(\alpha_{2}, \alpha_{1}\right)}{\wedge} h_{p} \stackrel{\left(\alpha_{2}, \alpha_{1}\right)}{\wedge} g_{p} \leq f_{p} \stackrel{\left(\alpha_{2}, \alpha_{1}\right)}{\circ} h_{p} \stackrel{\left(\alpha_{2}, \alpha_{1}\right)}{\circ} g_{p}$ and $f_{n} \underset{\left(\beta_{2}, \beta_{1}\right)}{\vee} h_{n} \underset{\left(\beta_{2}, \beta_{1}\right)}{\vee} g_{n} \geq f_{n} \underset{\left(\beta_{2}, \beta_{1}\right)}{\circ} h_{n} \underset{\left(\beta_{2}, \beta_{1}\right)}{\circ} g_{n}$ for every $\left(\alpha_{1}, \alpha_{2} ; \beta_{1}, \beta_{2}\right)$-BF right ideal $f=\left(S ; f_{p}, f_{n}\right)$, every $\left(\alpha_{1}, \alpha_{2} ; \beta_{1}, \beta_{2}\right)$-BF generalized bi-ideal $h=\left(S ; h_{p}, h_{n}\right)$ and every $\left(\alpha_{1}, \alpha_{2} ; \beta_{1}, \beta_{2}\right)$-BF left ideal $g=\left(S ; g_{p}, g_{n}\right)$ on $S$.

(3) $f_{p} \stackrel{\left(\alpha_{2}, \alpha_{1}\right)}{\wedge} h_{p} \stackrel{\left(\alpha_{2}, \alpha_{1}\right)}{\wedge} g_{p} \leq f_{p} \stackrel{\left(\alpha_{2}, \alpha_{1}\right)}{\circ} h_{p} \stackrel{\left(\alpha_{2}, \alpha_{1}\right)}{\circ} g_{p}$ and $f_{n} \underset{\left(\beta_{2}, \beta_{1}\right)}{\vee} h_{n} \underset{\left(\beta_{2}, \beta_{1}\right)}{\vee} g_{n} \geq f_{n} \underset{\left(\beta_{2}, \beta_{1}\right)}{\circ} h_{n} \underset{\left(\beta_{2}, \beta_{1}\right)}{\circ} g_{n}$ for every $\left(\alpha_{1}, \alpha_{2} ; \beta_{1}, \beta_{2}\right)$-BF right ideal $f=\left(S ; f_{p}, f_{n}\right)$, every $\left(\alpha_{1}, \alpha_{2} ; \beta_{1}, \beta_{2}\right)$-BF bi-ideal $h=\left(S ; h_{p}, h_{n}\right)$ and every $\left(\alpha_{1}, \alpha_{2} ; \beta_{1}, \beta_{2}\right)$-BF left ideal $g=\left(S ; g_{p}, g_{n}\right)$ on $S$.

(4) $f_{p} \stackrel{\left(\alpha_{2}, \alpha_{1}\right)}{\wedge} h_{p} \stackrel{\left(\alpha_{2}, \alpha_{1}\right)}{\wedge} g_{p} \leq f_{p} \stackrel{\left(\alpha_{2}, \alpha_{1}\right)}{\circ} h_{p} \stackrel{\left(\alpha_{2}, \alpha_{1}\right)}{\circ} g_{p}$ and $f_{n} \underset{\left(\beta_{2}, \beta_{1}\right)}{\vee} h_{n} \underset{\left(\beta_{2}, \beta_{1}\right)}{\vee} g_{n} \geq f_{n} \underset{\left(\beta_{2}, \beta_{1}\right)}{\circ} h_{n} \underset{\left(\beta_{2}, \beta_{1}\right)}{\circ} g_{n}$ for every $\left(\alpha_{1}, \alpha_{2} ; \beta_{1}, \beta_{2}\right)$-BF right ideal $f=\left(S ; f_{p}, f_{n}\right)$, every $\left(\alpha_{1}, \alpha_{2} ; \beta_{1}, \beta_{2}\right)$-BF quasi-ideal $h=\left(S ; h_{p}, h_{n}\right)$ and every $\left(\alpha_{1}, \alpha_{2} ; \beta_{1}, \beta_{2}\right)$-BF left ideal $g=\left(S ; g_{p}, g_{n}\right)$ on $S$.

Proof. $(1 \Rightarrow 2)$. Let $f, h$ and $g$ be a $\left(\alpha_{1}, \alpha_{2} ; \beta_{1}, \beta_{2}\right)$-BF right ideal, a $\left(\alpha_{1}, \alpha_{2} ; \beta_{1}, \beta_{2}\right)$-BF generalized bi-ideal and a $\left(\alpha_{1}, \alpha_{2} ; \beta_{1}, \beta_{2}\right)$-BF left ideal on $S$, respectively. Let $a \in S$. Because $S$ is regular, there exists $x \in S$ such that $a=$ axa. Thus

$$
\begin{aligned}
\left(f_{p} \stackrel{\left(\alpha_{2}, \alpha_{1}\right)}{\circ} h_{p} \stackrel{\left(\alpha_{2}, \alpha_{1}\right)}{\circ} g_{p}\right)(a) & =\left(\left(f_{p} \bar{o} h_{p} \bar{\sigma} g_{p}\right)(a) \wedge \alpha_{2}\right) \vee \alpha_{1} \\
& =\left(\bigvee_{a=y z}\left\{f_{p}(y) \wedge\left(h_{p} \bar{\sigma} g_{p}\right)(z) \wedge \alpha_{2}\right\}\right) \vee \alpha_{1} \\
& \geq\left(f_{p}(a x) \wedge\left(h_{p} \bar{\sigma} g_{p}\right)(a) \wedge \alpha_{2}\right) \vee \alpha_{1} \\
& \geq\left(f_{p}(a x) \vee \alpha_{1}\right) \wedge\left(\left(h_{p} \bar{\sigma} g_{p}\right)(a) \vee \alpha_{1}\right) \wedge\left(\alpha_{2} \vee \alpha_{1}\right) \\
& \geq\left(f_{p}(a) \wedge \alpha_{2}\right) \wedge\left(\bigvee_{a=r s}\left\{\left(h_{p}(r) \wedge g_{p}(s)\right) \vee \alpha_{1}\right\}\right) \wedge\left(\alpha_{2} \vee \alpha_{1}\right) \\
& \geq\left(f_{p}(a) \wedge \alpha_{2}\right) \wedge\left(\left(h_{p}(a) \vee \alpha_{1}\right) \wedge\left(g_{p}(x a) \vee \alpha_{1}\right) \wedge\left(\alpha_{2} \vee \alpha_{1}\right)\right. \\
& \geq\left(f_{p}(a) \wedge \alpha_{2}\right) \wedge\left(\left(h_{p}(a) \vee \alpha_{1}\right) \wedge\left(\left(g_{p}(a) \wedge \alpha_{2}\right) \vee \alpha_{1}\right) \wedge\left(\alpha_{2} \vee \alpha_{1}\right)\right. \\
& \geq\left(f_{p}(a) \wedge h_{p}(a) \wedge g_{p}(a) \wedge \alpha_{2}\right) \wedge \alpha_{1} \\
& =\left(\left(f_{p} \wedge h_{p} \wedge g_{p}\right)(a) \wedge \alpha_{2}\right) \wedge \alpha_{1} \\
& =\left(f_{p}\left(\alpha_{2}, \alpha_{1}\right) h_{p}{ }^{\left(\alpha_{2}, \alpha_{1}\right)} g_{p}\right)(a)
\end{aligned}
$$

Similarly, we can show that $f_{n} \underset{\left(\beta_{2}, \beta_{1}\right)}{\vee} h_{n} \underset{\left(\beta_{2}, \beta_{1}\right)}{\vee} g_{n} \geq f_{n} \underset{\left(\beta_{2}, \beta_{1}\right)}{\circ} h_{n} \underset{\left(\beta_{2}, \beta_{1}\right)}{\circ} g_{n}$.

$(2 \Rightarrow 3 \Rightarrow 4)$. This is straightforward, because every $\left(\alpha_{1}, \alpha_{2} ; \beta_{1}, \beta_{2}\right)$-BF bi-ideal is a $\left(\alpha_{1}, \alpha_{2} ; \beta_{1}, \beta_{2}\right)$-BF generalized bi-ideal and every $\left(\alpha_{1}, \alpha_{2} ; \beta_{1}, \beta_{2}\right)$-BF quasi-ideal is a $\left(\alpha_{1}, \alpha_{2} ; \beta_{1}, \beta_{2}\right)$-BF bi-ideal on $S$. 
$(4 \Rightarrow 1)$. Let $f$ and $g$ be any $\left(\alpha_{1}, \alpha_{2} ; \beta_{1}, \beta_{2}\right)$-BF right ideal and $\left(\alpha_{1}, \alpha_{2} ; \beta_{1}, \beta_{2}\right)$-BF left ideal on $S$, respectively. Let $a \in S$. By Theorem $8, \mathcal{S}=\left(S, \mathcal{S}_{p}, \mathcal{S}_{n}\right)$ is a $\left(\alpha_{1}, \alpha_{2} ; \beta_{1}, \beta_{2}\right)$-BF quasi ideal, and we have

$$
\begin{aligned}
\left(f_{p} \stackrel{\left(\alpha_{2}, \alpha_{1}\right)}{\wedge} g_{p}\right)(a) & =\left(\left(f_{p} \wedge g_{p}\right)(a) \wedge \alpha_{2}\right) \vee \alpha_{1} \\
& =\left(\left(f_{p} \wedge \mathcal{S}_{p} \wedge g_{p}\right)(a) \wedge \alpha_{2}\right) \vee \alpha_{1} \\
& =\left(f_{p} \stackrel{\left(\alpha_{2}, \alpha_{1}\right)}{\wedge} \mathcal{S}_{p} \stackrel{\left(\alpha_{2}, \alpha_{1}\right)}{\wedge} g_{p}\right)(a) \\
& \leq\left(f_{p} \stackrel{\left(\alpha_{2}, \alpha_{1}\right)}{\circ} \mathcal{S}_{p} \stackrel{\left(\alpha_{2}, \alpha_{1}\right)}{\circ} g_{p}\right)(a) \\
& \leq\left(f_{p} \stackrel{\left(\alpha_{2}, \alpha_{1}\right)}{\circ} g_{p}\right)(a)
\end{aligned}
$$

Thus $f_{p} \stackrel{\left(\alpha_{2}, \alpha_{1}\right)}{\wedge} g_{p} \leq f_{p}{ }^{\left(\alpha_{2}, \alpha_{1}\right)} g_{p}$ for every $\left(\alpha_{1}, \alpha_{2} ; \beta_{1}, \beta_{2}\right)$-BF right ideal $f$ and every $\left(\alpha_{1}, \alpha_{2} ; \beta_{1}, \beta_{2}\right)$-BF left ideal $g$ on $S$. Similarly, we can show that $f_{n} \underset{\left(\beta_{2}, \beta_{1}\right)}{\circ} g_{n} \leq f_{n} \underset{\left(\beta_{2}, \beta_{1}\right)}{\vee} g_{n}$. By Lemma 4, $f_{p} \stackrel{\left(\alpha_{2}, \alpha_{1}\right)}{\circ} g_{p} \leq f_{p} \stackrel{\left(\alpha_{2}, \alpha_{1}\right)}{\wedge} g_{p}$ and $f_{n} \underset{\left(\beta_{2}, \beta_{1}\right)}{\circ} g_{n} \geq f_{n} \underset{\left(\beta_{2}, \beta_{1}\right)}{\vee} g_{n}$. Thus $f_{p} \stackrel{\left(\alpha_{2}, \alpha_{1}\right)}{\circ} g_{p}=f_{p} \stackrel{\left(\alpha_{2}, \alpha_{1}\right)}{\wedge} g_{p}$ and $f_{n} \underset{\left(\beta_{2}, \beta_{1}\right)}{\circ} g_{n}=f_{n} \underset{\left(\beta_{2}, \beta_{1}\right)}{\vee} g_{n}$. Therefore by Theorem 9, $S$ is regular.

Theorem 11. For a semigroup $S$, the following are equivalent.

(1) $S$ is regular.

(2) $f_{p}^{\left(\alpha_{2}, \alpha_{1}\right)}=f_{p} \stackrel{\left(\alpha_{2}, \alpha_{1}\right)}{\circ} \mathcal{S}_{p} \stackrel{\left(\alpha_{2}, \alpha_{1}\right)}{\circ} f_{p}$ and $f_{n}^{\left(\beta_{2}, \beta_{1}\right)}=f_{n} \underset{\left(\beta_{2}, \beta_{1}\right)}{\circ} \mathcal{S}_{n} \underset{\left(\beta_{2}, \beta_{1}\right)}{\circ} f_{n}$ for every $\left(\alpha_{1}, \alpha_{2} ; \beta_{1}, \beta_{2}\right)-B F$ generalized bi-ideal $f=\left(S ; f_{p}, f_{n}\right)$ on $S$.

(3) $f_{p}^{\left(\alpha_{2}, \alpha_{1}\right)}=f_{p} \stackrel{\left(\alpha_{2}, \alpha_{1}\right)}{\circ} \mathcal{S}_{p} \stackrel{\left(\alpha_{2}, \alpha_{1}\right)}{\circ} f_{p}$ and $f_{n}^{\left(\beta_{2}, \beta_{1}\right)}=f_{n} \underset{\left(\beta_{2}, \beta_{1}\right)}{\circ} \mathcal{S}_{n} \underset{\left(\beta_{2}, \beta_{1}\right)}{\circ} f_{n}$ for every $\left(\alpha_{1}, \alpha_{2} ; \beta_{1}, \beta_{2}\right)$-BF bi-ideal $f=\left(S ; f_{p}, f_{n}\right)$ on $S$.

(4) $f_{p}^{\left(\alpha_{2}, \alpha_{1}\right)}=f_{p} \stackrel{\left(\alpha_{2}, \alpha_{1}\right)}{\circ} \mathcal{S}_{p} \stackrel{\left(\alpha_{2}, \alpha_{1}\right)}{\circ} f_{p}$ and $f_{n}^{\left(\beta_{2}, \beta_{1}\right)}=f_{n} \underset{\left(\beta_{2}, \beta_{1}\right)}{\circ} \mathcal{S}_{n} \underset{\left(\beta_{2}, \beta_{1}\right)}{\circ} f_{n}$ for every $\left(\alpha_{1}, \alpha_{2} ; \beta_{1}, \beta_{2}\right)$-BF quasi-ideal $f=\left(S ; f_{p}, f_{n}\right)$ on $S$.

Proof. $(1 \Rightarrow 2)$. Let $f$ be a $\left(\alpha_{1}, \alpha_{2} ; \beta_{1}, \beta_{2}\right)$-BF generalized bi-ideal on $S$ and $a \in S$. Because $S$ is regular, there exists $x \in S$ such that $a=a x a$. Hence we have

$$
\begin{aligned}
\left(f_{p}^{\left(\alpha_{2}, \alpha_{1}\right)} \mathcal{S}_{p} \stackrel{\left(\alpha_{2}, \alpha_{1}\right)}{\circ} f_{p}\right)(a) & =\left(\left(f_{p} \overline{0} \mathcal{S}_{p} \overline{0} f_{p}\right)(a) \wedge \alpha_{2}\right) \vee \alpha_{1} \\
& =\left(\bigvee_{a=y z}\left\{\left(f_{p} \bar{\circ} \mathcal{S}_{p}\right)(y) \wedge f_{p}(z)\right\} \wedge \alpha_{2}\right) \vee \alpha_{1} \\
& \left.\geq\left(\left(f_{p} \overline{\bar{O}} \mathcal{S}_{p}\right)(a x) \wedge f_{p}(a)\right) \wedge \alpha_{2}\right) \vee \alpha_{1} \\
& \left.=\left(\left(\bigvee_{a x=r s}\left\{f_{p}(r) \wedge \mathcal{S}_{p}(s)\right\}\right) \wedge f_{p}(a)\right) \wedge \alpha_{2}\right) \vee \alpha_{1} \\
& \geq\left(\left(\left(f_{p}(a) \wedge \mathcal{S}_{p}(x)\right) \wedge f_{p}(a)\right) \wedge \alpha_{2}\right) \vee \alpha_{1} \\
& \left.\left.=\left(\left(f_{p}(a) \wedge 1\right)\right) \wedge f_{p}(a)\right) \wedge \alpha_{2}\right) \vee \alpha_{1} \\
& =\left(f_{p}(a) \wedge \alpha_{2}\right) \vee \alpha_{1} \\
& =f_{p}^{\left(\alpha_{2}, \alpha_{1}\right)}(a)
\end{aligned}
$$


Thus $f_{p} \stackrel{\left(\alpha_{2}, \alpha_{1}\right)}{\circ} \mathcal{S}_{p} \stackrel{\left(\alpha_{2}, \alpha_{1}\right)}{\circ} f_{p} \geq f_{p}^{\left(\alpha_{2}, \alpha_{1}\right)}$. Similarly, we can show that $f_{n} \underset{\left(\beta_{2}, \beta_{1}\right)}{\circ} \mathcal{S}_{n} \underset{\left(\beta_{2}, \beta_{1}\right)}{\circ} f_{n} \leq f_{n}^{\left(\beta_{2}, \beta_{1}\right)}$. By Theorem 3, $f_{p} \stackrel{\left(\alpha_{2}, \alpha_{1}\right)}{\circ} \mathcal{S}_{p} \stackrel{\left(\alpha_{2}, \alpha_{1}\right)}{\circ} f_{p} \leq f_{p}^{\left(\alpha_{2}, \alpha_{1}\right)}$ and $f_{n} \underset{\left(\beta_{2}, \beta_{1}\right)}{\circ} \mathcal{S}_{n} \underset{\left(\beta_{2}, \beta_{1}\right)}{\circ} f_{n} \geq f_{n}^{\left(\beta_{2}, \beta_{1}\right)}$. Therefore, $f_{p} \stackrel{\left(\alpha_{2}, \alpha_{1}\right)}{\circ}$ $\mathcal{S}_{p} \stackrel{\left(\alpha_{2}, \alpha_{1}\right)}{\circ} f_{p}=f_{p}^{\left(\alpha_{2}, \alpha_{1}\right)}$ and $f_{n} \underset{\left(\beta_{2}, \beta_{1}\right)}{\circ} \mathcal{S}_{n} \underset{\left(\beta_{2}, \beta_{1}\right)}{\circ} f_{n}=f_{n}^{\left(\beta_{2}, \beta_{1}\right)}$.

$(2 \Rightarrow 3 \Rightarrow 4)$. Obvious.

$(4 \Rightarrow 1)$. Let $Q$ be any quasi-ideal of $S$. By Theorem 6 and Lemma 3, we have

$$
\begin{aligned}
\left(C_{Q}\right)_{p}^{\left(\alpha_{2}, \alpha_{1}\right)} & =\left(C_{Q}\right)_{p} \stackrel{\left(\alpha_{2}, \alpha_{1}\right)}{O}(\mathcal{S})_{p} \stackrel{\left(\alpha_{2}, \alpha_{1}\right)}{\circ}\left(C_{Q}\right)_{p} \\
& =\left(C_{Q S Q}\right)_{p}^{\left(\alpha_{2}, \alpha_{1}\right)}
\end{aligned}
$$

Thus, $Q=Q S Q$. Therefore it follows from Theorem 1 that $S$ is regular.

Theorem 12. For a semigroup $S$, the following are equivalent.

(1) $S$ is regular.

(2) $f_{p} \stackrel{\left(\alpha_{2}, \alpha_{1}\right)}{\wedge} g_{p} \leq f_{p} \stackrel{\left(\alpha_{2}, \alpha_{1}\right)}{\circ} g_{p}$ and $f_{n} \underset{\left(\beta_{2}, \beta_{1}\right)}{\vee} g_{n} \geq f_{n} \underset{\left(\beta_{2}, \beta_{1}\right)}{\circ} g_{n}$ for every $\left(\alpha_{1}, \alpha_{2} ; \beta_{1}, \beta_{2}\right)$-BF generalized bi-ideal $f=\left(S ; f_{p}, f_{n}\right)$ and every $\left(\alpha_{1}, \alpha_{2} ; \beta_{1}, \beta_{2}\right)$-BF left ideal $g=\left(S ; g_{p}, g_{n}\right)$ on $S$.

(3) $f_{p} \stackrel{\left(\alpha_{2}, \alpha_{1}\right)}{\wedge} g_{p} \leq f_{p} \stackrel{\left(\alpha_{2}, \alpha_{1}\right)}{\circ} g_{p}$ and $f_{n} \underset{\left(\beta_{2}, \beta_{1}\right)}{\vee} g_{n} \geq f_{n} \underset{\left(\beta_{2}, \beta_{1}\right)}{\circ} g_{n}$ for every $\left(\alpha_{1}, \alpha_{2} ; \beta_{1}, \beta_{2}\right)$-BF bi-ideal $f=\left(S ; f_{p}, f_{n}\right)$ and every $\left(\alpha_{1}, \alpha_{2} ; \beta_{1}, \beta_{2}\right)$-BF left ideal $g=\left(S ; g_{p}, g_{n}\right)$ on $S$.

(4) $f_{p} \stackrel{\left(\alpha_{2}, \alpha_{1}\right)}{\wedge} g_{p} \leq f_{p} \stackrel{\left(\alpha_{2}, \alpha_{1}\right)}{\circ} g_{p}$ and $f_{n} \underset{\left(\beta_{2}, \beta_{1}\right)}{\vee} g_{n} \geq f_{n} \underset{\left(\beta_{2}, \beta_{1}\right)}{\circ} g_{n}$ for every $\left(\alpha_{1}, \alpha_{2} ; \beta_{1}, \beta_{2}\right)$-BF quasi-ideal $f=\left(S ; f_{p}, f_{n}\right)$ and every $\left(\alpha_{1}, \alpha_{2} ; \beta_{1}, \beta_{2}\right)$-BF left ideal $g=\left(S ; g_{p}, g_{n}\right)$ on $S$.

Proof. $(1 \Rightarrow 2)$. Let $f$ and $g$ be any $\left(\alpha_{1}, \alpha_{2} ; \beta_{1}, \beta_{2}\right)$-BF generalized bi-ideal and any $\left(\alpha_{1}, \alpha_{2} ; \beta_{1}, \beta_{2}\right)$-BF left ideal on $S$, respectively. Let $a \in S$. Because $S$ is regular, there exists $x \in S$ such that $a=a x a$. Thus we have

$$
\begin{aligned}
\left(f_{p} \stackrel{\left(\alpha_{2}, \alpha_{1}\right)}{\circ} g_{p}\right)(a) & =\left(\left(f_{p} \bar{\sigma} g_{p}\right)(a) \wedge \alpha_{2}\right) \vee \alpha_{1} \\
& =\left(\bigvee_{a=y z}\left\{f_{p}(y) \wedge g_{p}(z)\right\} \wedge \alpha_{2}\right) \vee \alpha_{1} \\
& \left.\geq\left(f_{p}(a) \wedge g_{p}(x a)\right) \wedge \alpha_{2}\right) \vee \alpha_{1} \\
& \geq\left(f_{p}(a) \vee \alpha_{1}\right) \wedge\left(g_{p}(x a) \vee \alpha_{1}\right) \wedge\left(\alpha_{2} \vee \alpha_{1}\right) \\
& \geq\left(f_{p}(a) \vee \alpha_{1}\right) \wedge\left(\left(g_{p}(a) \wedge \alpha_{2}\right) \vee \alpha_{1}\right) \wedge\left(\alpha_{2} \vee \alpha_{1}\right) \\
& =\left(f_{p}(a) \wedge g_{p}(a) \wedge \alpha_{2}\right) \vee \alpha_{1} \\
& =\left(\left(f_{p} \wedge g_{p}\right)(a) \wedge \alpha_{2}\right) \vee \alpha_{1} \\
& =\left(f_{p} \stackrel{\left(\alpha_{2}, \alpha_{1}\right)}{\wedge} g_{p}\right)(a)
\end{aligned}
$$

Hence $f_{p} \stackrel{\left(\alpha_{2}, \alpha_{1}\right)}{\circ} g_{p} \geq f_{p} \stackrel{\left(\alpha_{2}, \alpha_{1}\right)}{\wedge} g_{p}$. Similarly, we can show that $f_{n} \underset{\left(\beta_{2}, \beta_{1}\right)}{\circ} g_{n} \leq f_{n} \underset{\left(\beta_{2}, \beta_{1}\right)}{\vee} g_{n}$.

$(2 \Rightarrow 3 \Rightarrow 4)$. Obvious.

$(4 \Rightarrow 1)$. Let $f$ and $g$ be any $\left(\alpha_{1}, \alpha_{2} ; \beta_{1}, \beta_{2}\right)$-BF right ideal and $\left(\alpha_{1}, \alpha_{2} ; \beta_{1}, \beta_{2}\right)$-BF left ideal on $S$, respectively. By Theorem $8, f$ is a $\left(\alpha_{1}, \alpha_{2} ; \beta_{1}, \beta_{2}\right)$-BF quasi ideal. Thus $f_{p} \stackrel{\left(\alpha_{2}, \alpha_{1}\right)}{\circ} g_{p} \geq f_{p} \stackrel{\left(\alpha_{2}, \alpha_{1}\right)}{\wedge} g_{p}$ 
and $f_{n} \underset{\left(\beta_{2}, \beta_{1}\right)}{\circ} g_{n} \leq f_{n} \underset{\left(\beta_{2}, \beta_{1}\right)}{\vee} g_{n}$. By Lemma $4, f_{p} \stackrel{\left(\alpha_{2}, \alpha_{1}\right)}{\circ} g_{p} \leq f_{p} \stackrel{\left(\alpha_{2}, \alpha_{1}\right)}{\wedge} g_{p}$ and $f_{n} \underset{\left(\beta_{2}, \beta_{1}\right)}{\circ} g_{n} \geq f_{n} \underset{\left(\beta_{2}, \beta_{1}\right)}{\vee} g_{n}$. Thus $f_{p} \stackrel{\left(\alpha_{2}, \alpha_{1}\right)}{\circ} g_{p}=f_{p} \stackrel{\left(\alpha_{2}, \alpha_{1}\right)}{\wedge} g_{p}$ and $f_{n} \underset{\left(\beta_{2}, \beta_{1}\right)}{\circ} g_{n}=f_{n} \underset{\left(\beta_{2}, \beta_{1}\right)}{\vee} g_{n}$. Therefore by Theorem 9, $S$ is regular.

\section{Conclusions}

In this paper, we propose the generalizations of BF sets. In particular, we introduce several concepts of generalized BF sets and study the relationship between such sets and semigroups. In other words, we propose generalized BF subsemigroups. This under consideration, the results obtained in this paper are some inequalities of $\left(\alpha_{1}, \alpha_{2} ; \beta_{1}, \beta_{2}\right)$-BF quasi(generalized bi-, bi-) ideals and characterize a regular semigroup in terms of generalized BF semigroups. The importance of BF sets has positive and negative components frequently found in daily life, for example, in organizations, economics, performance, development, evaluation, risk management or decisions, and so forth. Therefore we establish generalized BF sets on semigroups, which enhances the structure of the algebra. We hope that the study of some types of subsemigroups characterized in terms of inequalities of generalized BF subsemigroups is a useful mathematical tool.

Author Contributions: Both authors contributed equally to this manuscript.

Conflicts of Interest: The authors declare no conflict of interest.

\section{References}

1. Bloch, I. Bipolar Fuzzy Mathematical Morphology for Spatial Reasoning. Int. J. Approx. Reason. 2012, 53, 1031-1070.

2. Zadeh, L.A. Fuzzy sets. Inf. Control 1965, 8, 338-353.

3. Bucolo, M.; Fazzino, S.; La Rosa, M.; Fortuna, L. Small-world networks of fuzzy chaotic oscillators. Chaos Solitons Fractals 2003, 17, 557-565.

4. Rosenfeld, A. Fuzzy group. J. Math. Anal. Appl. 1971, 35, 338-353.

5. Kuroki, N. On fuzzy ideals and fuzzy bi-ideals in semigroups. Fuzzy Sets Syst. 1981, 5, 203-215.

6. Zhang, W.R. Bipolar Fuzzy Sets and Relations: A Computational Framework Forcognitive Modeling and Multiagent Decision Analysis. In Proceedings of the 1994 Industrial Fuzzy Control and Intelligent Systems Conference and the NASA Joint Technology Workshop on Neural Networks and Fuzzy Logic Fuzzy Information Processing Society Biannual Conference, San Antonio, TX, USA, 18-21 December 1994; pp. 305-309.

7. Lee, K.M. Bipolar-Valued Fuzzy Sets and Their Operations. In Proceedings of the International Conference on Intelligent Technologies, Bangkok, Thailand, 13-15 December 2000; pp. 307-312.

8. Kim, C.S.; Kang, J.G.; Kang, J.M. Ideal theory of semigroups based on the bipolar valued fuzzy set theory. Ann. Fuzzy Math. Inform. 2011, 2, 193-206.

9. Mordeson, J.N.; Malik, D.S.; Kuroki, N. Fuzzy Semigroups; Springer: Berlin, Germany, 2003.

(c) 2017 by the authors. Licensee MDPI, Basel, Switzerland. This article is an open access article distributed under the terms and conditions of the Creative Commons Attribution (CC BY) license (http://creativecommons.org/licenses/by/4.0/). 